
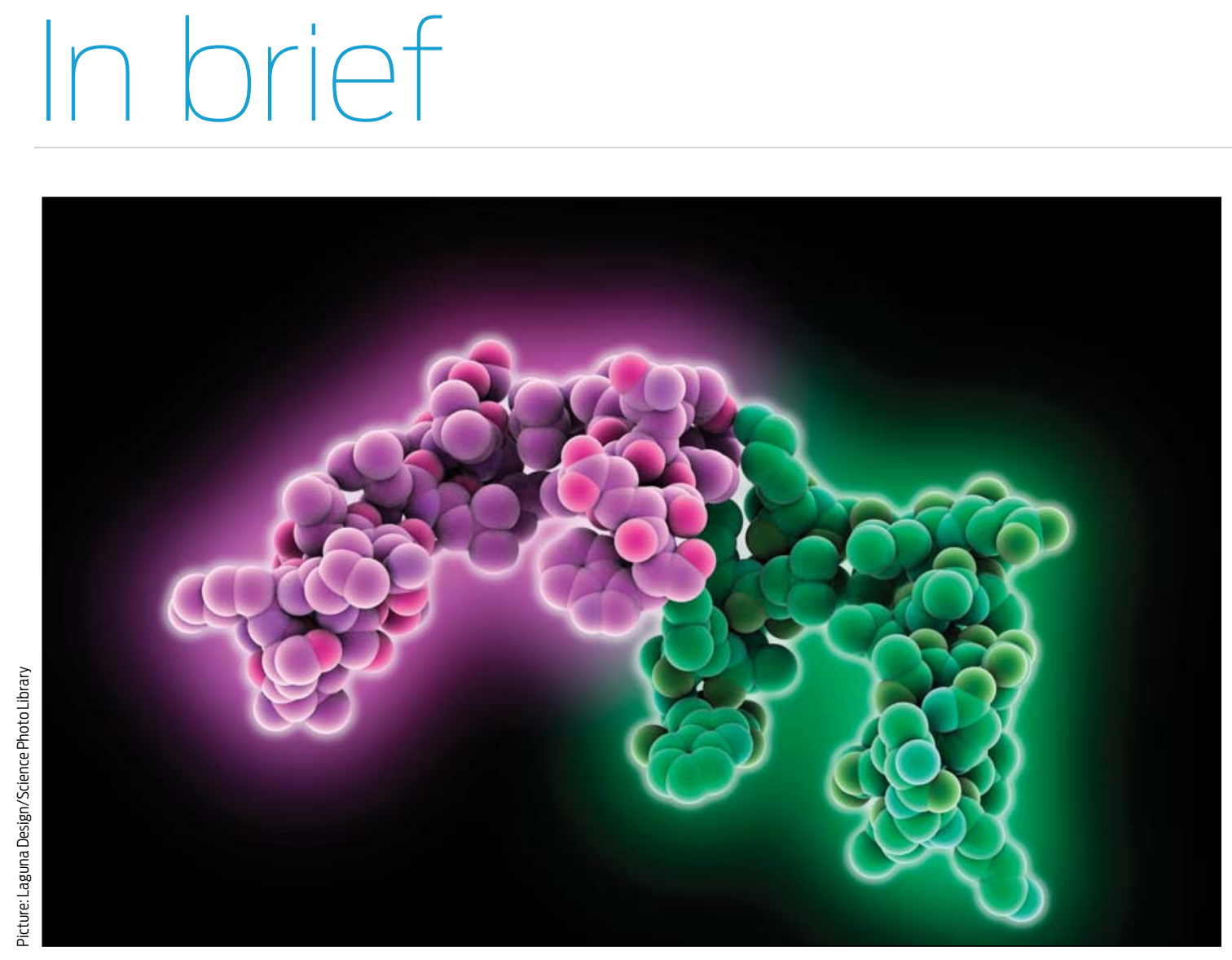

A model of the transcription factor hepatocyte nuclear factor-1 alpha (HNF1A). Transcription factors are proteins that bind to specific sequences of DNA and control the transcription of genetic information from DNA to RNA. HNF1A is predominantly expressed in the liver where it is involved in regulating insulin expression and glucose metabolism. Mutations in this protein cause a form of type 2 diabetes.

\title{
From The Cochrane Library
}

\section{Cochrane reviews - now available in convenient daily doses}

In a sign of the times, The Cochrane Library has recently switched from monthly publication to a continuous publishing model — proof that good news comes even to those who can't wait. And reading the latest Cochrane reviews with a freshly brewed cup of tea could be just what the doctor ordered. According to a review of 11 studies, green or black tea may help to lower the risk of cardiovascular disease by reducing low-density lipoprotein cholesterol and blood pressure (doi: 10.1002/14651858.CD009934. pub2). Another review hints that the same could be true of increased consumption of fruits and vegetables (doi: 10.1002/14651858.CD009874. pub2). Both conclusions are tentative though, as neither review included studies with long-term outcomes, and many trials were small and of moderate or poor quality.

Obtaining informed consent for surgery or other procedures is a crucial part of patient care that, despite the best intentions, can lead to misunderstanding. A new review of 65 randomised trials of interventions designed to improve either the delivery or the consideration of information for informed consent found that they improved knowledge of the planned procedure (both short and long term) and reduced decisional conflict.

These findings hold true despite wide variations in the setting, interventions used and outcomes measured proof that pragmatic steps to improve informed consent are likely to benefit patients (doi: 10.1002/14651858. CD009445.pub2).

A timely review for winter found that paracetamol may help relieve nasal obstruction and rhinorrhoea but may be best left on the shelf for other symptoms of the common cold, such as sore throat, sneezing and coughing.

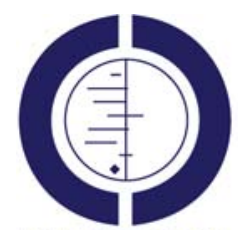

THE COCHRANE COLLABORATION $^{8}$

\section{Steve McDonald \\ Co-Director}

Tari Turner

Adjunct Senior Research Fellow

Australasian Cochrane Centre

doi: 10.5694/mjal3.10910
Since the evidence is inconclusive, perhaps our best comfort is knowing that winter is almost over (doi: 10.1002/14651858.CD008800.pub2).

We hear a lot about what makes a good death, and for many with advanced illness, their wish is to die at home. A sizeable review of 23 studies involving nearly 38000 participants, mostly with cancer, and 4000 family caregivers has found clear and reliable evidence that home palliative care services double the chance of being able to die at home and also reduce the overall symptom burden. Importantly, these gains are achieved without increasing the grief for family caregivers after the patient dies (doi: 10.1002/14651858.CD007760.pub2).

Remember to check out new reviews added daily to The Cochrane Library at www.thecochranelibrary.com. 


\section{Guidelines ignored in back pain management}

Management of back pain in the US relies on measures that are not supported by published professional guidelines, resulting in increased costs and overuse of imaging, says research in JAMA Internal Medicine. The study used data on 23918 visits for spine problems from the National Ambulatory Medical Care Survey and the National Hospital Ambulatory Medical Care Survey to characterise the treatment of back pain from 1 January 1999 to 26 December 2010.

The authors found that non-steroidal anti-inflammatory drug or paracetamol use (supported by guidelines) per visit decreased from $36.9 \%$ in $1999-2000$ to $24.5 \%$ in $2009-2010$, while narcotic use (unsupported) increased from $19.3 \%$ to $29.1 \%$. Physical therapy referrals (supported) stayed stable at approximately $20 \%$, but physician referrals (unsupported) increased from $6.9 \%$ to $14.0 \%$. Radiographs (supported) remained stable at approximately $17 \%$, but computed tomography scans and magnetic resonance imaging (unsupported) increased from $7.2 \%$ to $11.3 \%$. An accompanying editorial said: "[This study] forces us to admit that the development of clinical guidelines alone will not solve our problem in managing back pain."

JAMA Intern Med 2013; 29 July (online) doi: 10.1001/jamainternmed.2013.899 doi: 10.1001/jamainternmed.2013.7672

\section{Lower costs improve hypertension control}

A systematic review of 53 studies has found an association between reduced out-of-pocket patient costs and improved hypertension (HT) control and treatment adherence. The PLOS Medicine review also found that, in US settings, having health insurance and a routine physician or place of care were also associated with improved HT outcomes. The review set out to analyse the "influence of national or regional health systems on HT awareness, treatment and control". Databases searched were MEDLINE, Embase, Global Health, LILACS, Africa-Wide Information, IMSEAR, IMEMR and WPRIM with no date or language restrictions. Eleven of the 53 eligible studies were conducted in low- or middleincome countries. "This review supports the minimization of medication co-payments in health insurance plans, and although the studies were largely conducted in the US, the principle is likely to apply more generally", the authors wrote.

PLOS Medicine 2013; 30 July (online) doi: 10.1371/journal.pmed.1001490

\section{Proximity to benzene sites increases risk of $\mathrm{NHL}$}

An association has been found between distance from a benzene release point and the incidence of non-Hodgkin lymphoma (NHL), according to research published in Cancer. The authors linked and geocoded data on benzene release sites (such as manufacturing facilities, service businesses and government facilities) in the US state of Georgia from 1988 to 1998 using the Environmental Protection Agency's Toxics Release Inventory, census tract level population data from the 2000 census, and NHL incidence data from the Georgia Comprehensive Cancer Registry from 1999 to 2008. They found that for every mile the average distance to benzene release sites increased, there was an expected $0.31 \%$ decrease in the rate of NHL. In addition, "Clusters of NRL were spatially associated with benzene release sites located in metropolitan areas, but not with release sites in other areas of the state", the authors wrote. Cancer 2013; 29 July (online). doi: 10.1002/cncr.28083

\section{From the MJA archives}

\section{MJA 1935: 20 July (edited extract)}

\section{"Doctor"}

Sir: The well informed criticism in one of our powerful dailies raises an important point and deserves the attention of the Council of the British Medical Association. Is a medical practitioner who does not hold the M.D. entitled to call himself "Doctor"? Perhaps the public are largely to blame for the misuse of the word doctor. They persist in regarding every medical practitioner as a doctor and vice versa. Thus a doctor of laws or a doctor of philosophy, while at a holiday resort, may be urgently requested to attend to an accident case. The medical practitioner is primarily to blame for this, because of his prerogative claim to the distinguishing degree. Herbalists and osteopaths are forbidden by law to use the term doctor. A man with nothing more than M.B., B.S. is no more entitled than they to use the word as a prefix to his name. It is a false claim on his part, and he knows it.

"Physician", Collins Street, Melbourne

\section{Not all placebo effects are equal}

A crossover, two-experiment study published in PLOS One has found that individuals respond differently to different types of placebos, explaining why it has been difficult to define the subset of people who are "placebo responders". A significant difference was also found between genuine and sham acupuncture treatments in the study, implying "non-specific effects may significantly contribute to the analgesic effect observed in acupuncture analgesia". In the first experiment, 71 healthy subjects received one of four treatments: placebo pills (described as Tylenol), sham acupuncture, genuine acupuncture, or no treatment. Before and after each treatment, paired with positive verbal reinforcement, each subject's pain threshold, pain tolerance and pain ratings to calibrated heat ratings were measured. Fourteen days later, in experiment 2, subjects had their analgesic responses to conditioned visual cues tested. Forty-five subjects completed both experiments. The findings implied "that placebo responses may not be dependent on stable individual traits but rather are more a characteristic of the state circumstances of individuals or combination of both trait and state", the authors wrote.

PLOS One 2013; 31 July (online) doi: 10.1371/journal.pone.0067485 